\title{
Micro-Doppler Effect of Extended Streamlined Targets Based on Sliding Scattering Center Model
}

\author{
$B O T A N G$ \\ School of Computer and Communication Engineering, University of Science and Technology Beijing, \\ Xueyuanlu 30, Beijing, China \\ tangbo@ustb.edu.cn
}

Manuscript received December 7, 2015

\begin{abstract}
The scattering center of extended streamlined targets can slide when the direction of radiation is changed. The sliding scattering center has influence on the micro-Doppler effect of micro-motion of the extended streamlined target. This paper focused on the micro-Doppler of the extended streamlined target for the bistatic radar. Based on the analysis, the analytical expressions of the micro-Doppler of coning motion with sliding scattering center model were given for bistatic radar. And the results were validated by the simulated results of the scattering field based on the full-wave method of the electromagnetic computation. The results showed that the sliding of the scattering center can make the micro-Doppler be less and distorted, and the influence of the sliding is different for two different types of the sliding scattering centers: sliding on the surface and sliding on the bottom circle. The analytical expressions of the micro-Doppler are helpful to analyze the time-frequency presentations (TFR) of the coning motion of the extended streamlined target and to estimate the parameters of the target.
\end{abstract}

\section{Keywords}

Micro-Doppler, sliding scattering center, extended streamlined targets

\section{Introduction}

The micro-Doppler of the micro-motion of the radar target has been a hot point of researches for many years since V. C. Chen's work [1-3]. The characteristics of micro-Doppler caused by micro-motions are beneficial for target recognition, positioning and some other applications. The mass center of the target usually has a translational motion, and the micro-motion is the mechanical vibration and rotation of the target or the part of it [3]. Therefore, the micro-Doppler can reflect the dynamics features of the target. Because of the usefulness of the micro-Doppler in practice, a large quantity of researches on micro-Doppler has been made for more than ten years [4-7]. The research areas include the micro-Doppler of human bodies, the micro-Doppler of vehicles, and the micro-Doppler of flying targets and so on [8-10]. These researches mainly focused on what is the micro-Doppler effect corresponding to the micro-motion, how to obtain the characteristics of the micro-Doppler, and how to use it [11], [12].

The micro-Doppler of a target with mechanical vibration and rotation motions in addition to the translation motion of its mass center has been given for rigid bodies [3]. Not only the scattering centers have mechanical micro-motions, but also the scattering centers can have the electromagnetic micro-motion, which can be called scattering centers sliding. The influence of scattering centers sliding on the bistatic high range resolution profiles (HRRPs) was studied in [13], [14]. And the formation mechanism of sliding scattering centers was studied based on creeping waves theory in [15],[16]. In [17], the micro-Doppler of the cone-cylinder-shaped target with sliding scattering centers for the mono-static radar was studied. And in [17] there's only one type of the sliding scattering centers considered. In [18], the sliding scattering center model has been given and validated by the numerical computation results of the scattering field of the extended streamlined target. And the micro-Doppler effect of the extended streamlined target with sliding scattering centers has been studied in [18], [19]. But only micro-motions were considered in [18], [19], and the velocity of the translation motion was ignored. The micro-motion, translation motion, and the scattering center sliding, all these three kinds of motions can cause Doppler Effect. And the micro-Doppler is affected by the interactions of all these three kinds of motions. All these three kinds of motions should be considered in the micro-Doppler analysis for the extended streamlined target. Therefore, the micro-Doppler effect is studied in this paper based on the sliding scattering center model under the conditions of the translation motion and the cone motion for the bistatic radar.

This paper is organized as follows. Section 2 presents the motion model of the target for the bistatic radar; Section 3 gives the analytical expression of the micro-Doppler of the extended streamlined target; Section 4 gives the numerical results, and the simulated results based on the electromagnetic numerical computation are given to validate it. Finally, the conclusions are made. 


\section{Geometry Model of the Micro- motion of Extended Streamlined Targets}

The track of the mass center of the extended streamlined target is a straight line. The mass center is denoted as point M. And radar is located on the ground, denoted as points $\mathrm{A}$ and $\mathrm{B}$ for the receiver and the transmitter, respectively. The least distance between the receiver and the target is denoted as $R_{\mathrm{r}}$, and the corresponding point in the track line is point $\mathrm{O}$. The radar coordinates system $x y z$ is set as follows: the origin is $\mathrm{O}$, and $z$ axis is along the track line, and $x$ axis is from $\mathrm{O}$ point to the receiver. That is, the receiver coordinates in the radar coordinate system are $\left(R_{\mathrm{r}}\right.$, $0,0)$. The transmitter coordinates in the radar coordinate system are $\left(R_{\mathrm{t}} \cos \phi, R_{\mathrm{t}} \sin \phi, z_{\mathrm{t}}\right)$. In this paper, it is assumed that $R_{\mathrm{r}}>>|\mathrm{AB}|$ and $R_{\mathrm{t}}>>|\mathrm{AB}|$. The velocity amplitude of the mass center is denoted as $v$. It is set that the time $t=0$ when the mass center arrives at point $\mathrm{O}$. Thus, the coordinates of the mass center $\mathrm{M}$ are $(0,0, v t)$ as seen in Fig. 1.

The top point of the target is denoted as point $\mathrm{T}$. The angular velocity of the cone-motion of the target is $\omega$. And

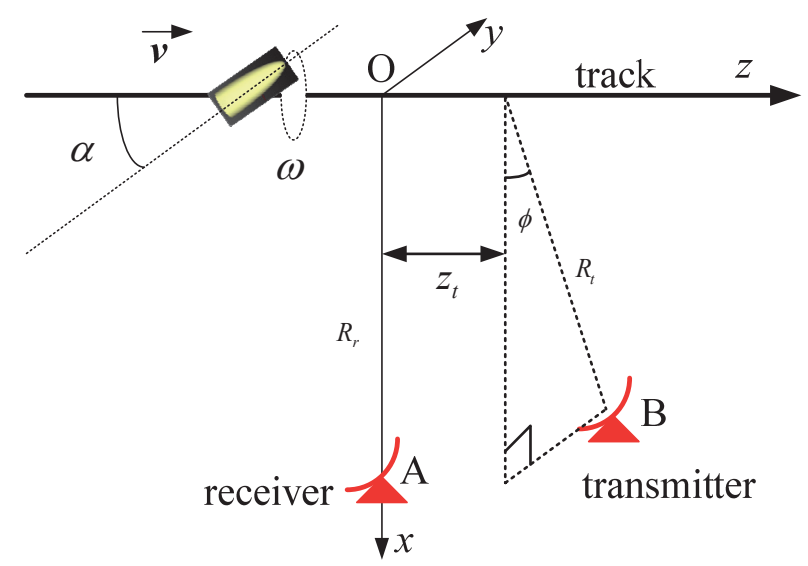

Fig. 1. Radar and the target in the radar coordinate system.

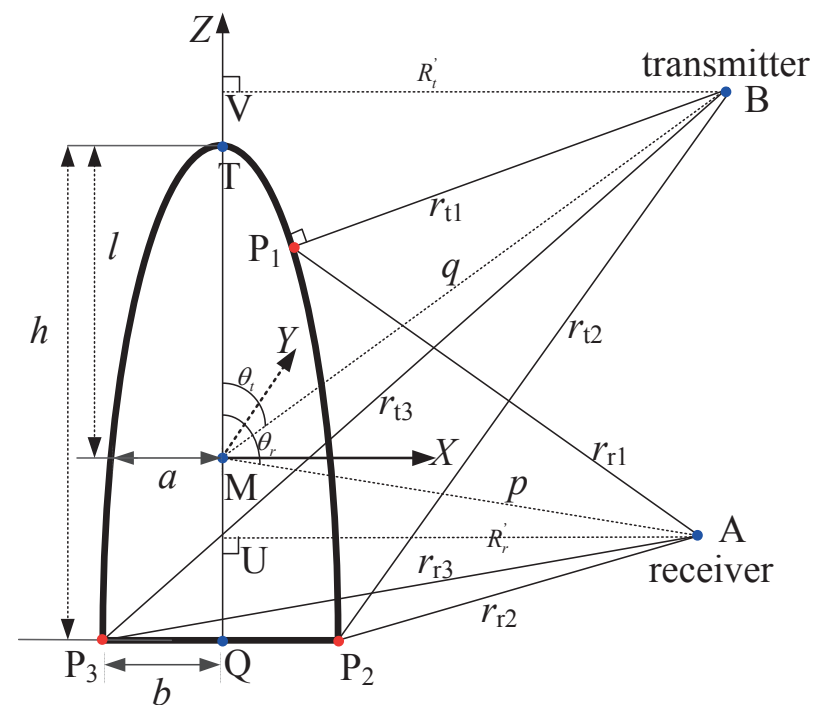

Fig. 2. Model in the target coordinates system (A is in $\mathrm{XZ}$ plane; B is not). the axis of the cone-motion is $\mathrm{z}$ axis. Then, the location of the top point $\mathrm{T}$ in the radar coordinates system is $\left(l \sin \alpha \sin \left(\omega t+\varphi_{0}\right), l \sin \alpha \cos \left(\omega t+\varphi_{0}\right), l \cos \alpha+v t\right)$ where $l$ is the distance between $\mathrm{M}$ and T. $\varphi_{0}$ is the original phase of the cone-motion.

The target coordinate system $X Y Z$ is set as follows. The plane determined by the receiver and the track line is defined as $X Z$ plane as seen in Fig. 2. The mass center $M$ is the origin of the $X Y Z$ coordinates system. And the $\mathrm{Z}$ axis is from point $M$ to $T$. The center of the bottom of the target is denoted as point $\mathrm{Q}$. The distance between $\mathrm{T}$ and $\mathrm{Q}$ is denoted as $h$. The radius of the cross section through $\mathrm{Q}$ is denoted as $b$, and the radius of the cross section through $\mathrm{M}$ is $a$. The distance from $\mathrm{A}$ and $\mathrm{B}$ to point $\mathrm{M}$ are denoted as $p$ and $q$ respectively. $\mathrm{U}$ is the nearest point in $Z$ axis to the receiver, and $\mathrm{V}$ is the nearest point in $Z$ axis to the transmitter.

According to the sliding scattering center model, the cone-shaped target will have three sliding scattering centers [18]. They are points $\mathrm{P}_{1}, \mathrm{P}_{2}$ and $\mathrm{P}_{3}$ respectively in Fig. 2 . The ranges from radar to these points are denoted as $r_{\mathrm{r} 1}, r_{\mathrm{r} 2}$, $r_{\mathrm{r} 3}, r_{\mathrm{t} 1}, r_{\mathrm{t} 2}$ and $r_{\mathrm{t} 3}$ respectively in Fig. 2.

Firstly, the triangle ATM is analyzed. Based on the locations of points $\mathrm{A}, \mathrm{T}$ and $\mathrm{M}$ in the radar coordinate system, the unit vector of $\overrightarrow{\mathrm{MA}}$ in the target coordinate system can be obtained as

$$
\begin{gathered}
\mathbf{e}_{\overline{\mathrm{MA}}}=\sin \theta_{\mathrm{r}} \mathbf{e}_{\mathrm{X}}+\cos \theta_{\mathrm{r}} \mathbf{e}_{\mathrm{Z}} \\
\theta_{r}=\arccos \frac{p^{2}+l^{2}-u^{2}}{2 p l}=\arccos \frac{R_{\mathrm{r}} \sin \alpha \sin \left(\omega t+\varphi_{0}\right)-v t \cos \alpha}{\sqrt{R_{r}^{2}+v^{2} t^{2}}} \\
p=\sqrt{R_{\mathrm{r}}^{2}+v^{2} t^{2}} \\
u=\mathrm{BT}=\sqrt{\left[R_{\mathrm{r}}-l \sin \alpha \sin \left(\omega t+\varphi_{0}\right)\right]^{2}+}
\end{gathered}
$$

In the same way, the triangle BTM is analyzed. Based on the locations of points $\mathrm{B}, \mathrm{T}$ and $\mathrm{M}$ in the radar coordinate system, the unit vector of $\overrightarrow{\mathrm{MB}}$ in the target coordinate system can be obtained as

$$
\begin{gathered}
\mathbf{e}_{\overline{\mathrm{MB}}}=\sin \theta_{\mathrm{t}} \cos \varphi_{\mathrm{t}} \mathbf{e}_{\mathrm{X}}+\sin \theta_{\mathrm{t}} \sin \varphi_{\mathrm{t}} \mathbf{e}_{\mathrm{Y}}+\cos \theta_{\mathrm{t}} \mathbf{e}_{\mathrm{Z}}, \\
\theta_{t}=\arccos \frac{q^{2}+l^{2}-s^{2}}{2 q l} \\
=\arccos \frac{R_{\mathrm{t}} \sin \alpha \sin \left(\omega t+\varphi_{0}+\phi\right)+\left(z_{\mathrm{t}}-v t\right) \cos \alpha}{\sqrt{R_{\mathrm{t}}^{2}+\left(z_{\mathrm{t}}-v t\right)^{2}}}, \\
s=\sqrt{R_{\mathrm{t}}^{2}+\left(z_{\mathrm{t}}-v t\right)^{2}}, \\
\left.\qquad R_{\mathrm{t}} \cos \phi-l \sin \alpha \sin \left(\omega t+\varphi_{0}\right)\right]^{2}+
\end{gathered}
$$


Let

$$
\begin{aligned}
w & =\left(\mathbf{e}_{\overline{\mathrm{MA}}} \times \mathbf{e}_{\overline{\mathrm{MT}}}\right) \cdot\left(\mathbf{e}_{\overline{\mathrm{MB}}} \times \mathbf{e}_{\overline{\mathrm{MT}}}\right)=\mathbf{e}_{\overline{\mathrm{MB}}} \cdot\left[\mathbf{e}_{\overline{\mathrm{MT}}} \times\left(\mathbf{e}_{\overline{\mathrm{MA}}} \times \mathbf{e}_{\overline{\mathrm{MT}}}\right)\right] \\
& =\mathbf{e}_{\overline{\mathrm{MB}}} \cdot\left[\left(\mathbf{e}_{\overline{\mathrm{MT}}} \cdot \mathbf{e}_{\overline{\mathrm{MT}}}\right) \mathbf{e}_{\overline{\mathrm{MA}}}-\left(\mathbf{e}_{\overline{\mathrm{MT}}} \cdot \mathbf{e}_{\overline{\mathrm{MA}}}\right) \mathbf{e}_{\overline{\mathrm{MT}}}\right] \\
& =\mathbf{e}_{\overline{\mathrm{MB}}} \cdot \mathbf{e}_{\overline{\mathrm{MA}}}-\left(\mathbf{e}_{\overline{\mathrm{MT}}} \cdot \mathbf{e}_{\overline{\mathrm{MA}}}\right)\left(\mathbf{e}_{\overline{\mathrm{MT}}} \cdot \mathbf{e}_{\overline{\mathrm{MB}}}\right) \\
& =\mathbf{e}_{\overline{\mathrm{MB}}} \cdot \mathbf{e}_{\overline{\mathrm{MA}}}-\cos \theta_{\mathrm{r}} \cos \theta_{\mathrm{t}}
\end{aligned}
$$

and $\quad \mathbf{e}_{\overline{\mathrm{MB}}} \cdot \mathbf{e}_{\overline{\mathrm{MA}}}=\frac{R_{\mathrm{r}} R_{\mathrm{t}} \cos \phi+(-v t)\left(z_{\mathrm{t}}-v t\right)}{p q}$.

Then

$$
\begin{gathered}
\cos \varphi_{\mathrm{t}}=\frac{w}{\sin \theta_{\mathrm{r}} \sin \theta_{\mathrm{t}}}=\frac{R_{\mathrm{r}} R_{\mathrm{t}} \cos \phi-v t\left(z_{\mathrm{t}}-v t\right)}{p q \sin \theta_{\mathrm{r}} \sin \theta_{\mathrm{t}}}-\cot \theta_{\mathrm{r}} \cot \theta_{\mathrm{t}} \\
\sin \varphi_{\mathrm{t}}=\eta \sqrt{1-\cos ^{2} \varphi_{\mathrm{t}}} \\
\eta=\frac{\sin \phi}{|\sin \phi|}
\end{gathered}
$$

$\mathrm{P}_{1}$ will slide on the surface in Fig. 2 with different radiation directions. Therefore, the calculation of $r_{\mathrm{t} 1}$ and $r_{\mathrm{r} 1}$ is a bit complicated. The location of $\mathrm{P}_{1}$ in the target coordinates system can be denoted as $\left(X_{1}, Y_{1}, Z_{1}\right)$. The target surface for $l-h \leq Z_{1} \leq l$ can be assumed to be a quadratic surface. Then,

$$
X_{1}^{2}+Y_{1}^{2}+c_{1} Z_{1}^{2}+c_{2} Z_{1}+c_{3}=0,\left(l-h \leq Z_{1} \leq l\right)
$$

where $c_{1} l^{2}+c_{2} l=a^{2}$ and $c_{3}=-a^{2}$. Assume $0 \leq c_{1} \leq 1$ for the target. The normal vector of the surface on $P_{1}$ is

$$
\mathbf{e}_{\mathrm{P}_{1}}=\left(2 X_{1}, 2 Y_{1}, 2 c_{1} Z_{1}+c_{2}\right) \text {. }
$$

The unit vector of the bisector of angle AMB is

$$
\begin{aligned}
& \mathbf{e}_{\mathrm{M}}=\left(\mathbf{e}_{\overline{\mathrm{MB}}}+\mathbf{e}_{\overline{\mathrm{MA}}}\right) / \tau \\
&= {\left[\begin{array}{c}
\left.\left(\sin \theta_{\mathrm{r}}+\sin \theta_{\mathrm{t}} \cos \varphi_{\mathrm{t}}\right) \mathbf{e}_{\mathrm{X}}+\sin \theta_{\mathrm{t}} \sin \varphi_{\mathrm{t}} \mathbf{e}_{\mathrm{Y}}+\right] / \tau, \\
\left(\cos \theta_{\mathrm{r}}+\cos \theta_{\mathrm{t}}\right) \mathbf{e}_{\mathrm{Z}}
\end{array}\right] } \\
& \tau=\left|\mathbf{e}_{\overline{\mathrm{MB}}}+\mathbf{e}_{\overline{\mathrm{MA}}}\right|
\end{aligned}
$$

when the distance between the radar and the target is far larger than the size of the target, $\mathbf{e}_{\mathrm{P} 1}$ should be parallel to $\mathbf{e}_{\mathrm{M}}$. Therefore,

$$
\frac{2 X_{1}}{\sin \theta_{\mathrm{r}}+\sin \theta_{\mathrm{t}} \cos \varphi_{\mathrm{t}}}=\frac{2 Y_{1}}{\sin \theta_{\mathrm{t}} \sin \varphi_{\mathrm{t}}}=\frac{2 c_{1} Z_{1}+c_{2}}{\cos \theta_{\mathrm{r}}+\cos \theta_{\mathrm{t}}} .
$$

The solution is

$X_{1}=\gamma\left(\sin \theta_{\mathrm{r}}+\sin \theta_{\mathrm{t}} \cos \varphi_{\mathrm{t}}\right)$,

$Y_{1}=\gamma \sin \theta_{\mathrm{t}} \sin \varphi_{\mathrm{t}}$,

$Z_{1}=\left\{\begin{array}{cc}-\frac{c_{2}}{2 c_{1}}+\frac{\gamma}{c_{1}}\left(\cos \theta_{\mathrm{r}}+\cos \theta_{\mathrm{t}}\right) & \text { for } \quad c_{1} \neq 0 \\ -\frac{c_{3}}{c_{2}}-\frac{\gamma^{2}}{c_{2}}\left(\sin ^{2} \theta_{\mathrm{r}}+\sin ^{2} \theta_{\mathrm{t}}+2 \sin \theta_{\mathrm{r}} \sin \theta_{\mathrm{t}} \cos \varphi_{\mathrm{t}}\right) & \text { for } c_{1}=0\end{array}\right.$

$$
\gamma=\sqrt{\frac{\frac{c_{2}^{2}}{4 c_{1}}-c_{3}}{\mu}}
$$

$\mu=2+\left(\frac{1}{c_{1}}-1\right)\left(\cos ^{2} \theta_{\mathrm{r}}+\cos ^{2} \theta_{\mathrm{t}}\right)+2 \sin \theta_{\mathrm{r}} \sin \theta_{\mathrm{t}} \cos \varphi_{t}+$

$$
\frac{2}{c_{1}} \cos \theta_{\mathrm{r}} \cos \theta_{\mathrm{t}} \text {. }
$$

Then,

$$
\Delta r_{1}=r_{r 1}+r_{t 1}-2 p=-\overrightarrow{\mathrm{OP}_{1}} \cdot\left(\mathbf{e}_{\overline{\mathrm{MB}}}+\mathbf{e}_{\overline{\mathrm{MA}}}\right) .
$$

Because the continuity of the physical phenomenon, the micro-Doppler under the situation $c_{1}=0$ is the limitation of the micro-Doppler under the situation $c_{1} \neq 0$. Therefore, only the situation $c_{1} \neq 0$ is considered in the following derivation.

Substitute (1), (5), (19) into (22), then it can be obtained that

$\Delta r_{1}=-\left[\begin{array}{c}\gamma\left(\sin \theta_{\mathrm{r}}+\sin \theta_{\mathrm{t}} \cos \varphi_{\mathrm{t}}\right)^{2}+\gamma\left(\sin \theta_{\mathrm{t}} \sin \varphi_{\mathrm{t}}\right)^{2}- \\ \frac{c_{2}}{2 c_{1}}\left(\cos \theta_{\mathrm{r}}+\cos \theta_{\mathrm{t}}\right)+\frac{\gamma}{c_{1}}\left(\cos \theta_{\mathrm{r}}+\cos \theta_{\mathrm{t}}\right)^{2}\end{array}\right]$.

Equation (23) can be rewritten as

$$
\Delta r_{1}=-\sqrt{\mu\left(\frac{c_{2}^{2}}{4 c_{1}}-c_{3}\right)}+\frac{c_{2}}{2 c_{1}}\left(\cos \theta_{\mathrm{r}}+\cos \theta_{\mathrm{t}}\right) .
$$

The location of $\mathrm{P}_{2}$ in the target coordinate system is $(b, 0, l-h)$. Therefore,

$$
\begin{aligned}
\Delta r_{2} & =r_{r 2}+r_{t 2}-2 p \cong-\overrightarrow{\mathrm{OP}_{2}} \cdot\left(\mathbf{e}_{\overline{\mathrm{MB}}}+\mathbf{e}_{\overline{\mathrm{MA}}}\right) \\
& =-b\left(\sin \theta_{\mathrm{r}}+\sin \theta_{\mathrm{t}} \cos \varphi_{\mathrm{t}}\right)-(l-h)\left(\cos \theta_{\mathrm{r}}+\cos \theta_{\mathrm{t}}\right) .
\end{aligned}
$$

And in the same way, it is obtained that

$$
\begin{aligned}
\Delta r_{3} & =r_{r 3}+r_{t 3}-2 p \cong-\overrightarrow{\mathrm{OP}_{3}} \cdot\left(\mathbf{e}_{\overline{\mathrm{MB}}}+\mathbf{e}_{\overline{\mathrm{MA}}}\right) \\
& =b\left(\sin \theta_{\mathrm{r}}+\sin \theta_{\mathrm{t}} \cos \varphi_{\mathrm{t}}\right)-(l-h)\left(\cos \theta_{\mathrm{r}}+\cos \theta_{\mathrm{t}}\right) .
\end{aligned}
$$

Although $\mathrm{P}_{2}$ and $\mathrm{P}_{3}$ are sliding points in three dimensions space, they are stationary in two dimensions $\mathrm{XZ}$ plane as seen in Fig. 2 with different radiation directions of radar.

\section{Micro-Doppler of the Extended Streamlined Target}

The Doppler frequency shift caused by the motion of $\mathrm{P}_{i}$ can be expressed as [3]

$$
f_{\mathrm{d}, 1}(t)=\frac{1}{2 \pi} \frac{\mathrm{d}}{\mathrm{d} t}\left(-k r_{i}\right), \quad(i=1,2,3)
$$

where $k$ is the wave number of the carrier frequency. 
The micro-Doppler caused by the micro-motion of $\mathrm{P}_{i}$ can be expressed as [3]

$$
f_{\mathrm{md}, 1}(t)=\frac{1}{2 \pi} \frac{\mathrm{d}}{\mathrm{d} t}\left(-k \Delta r_{i}\right) \quad(i=1,2,3)
$$

\section{a) Micro-Doppler of $P_{1}$}

Substituting (2), (6), (11), (20), (24) into (28), then equations (29) and (30) can be derived (see the bottom of this page).

Note that $\mathrm{P}_{1}$ exists only when $l-h \leq Z_{1} \leq l$. It can be seen that $f_{\mathrm{md}, 1}(t)$ is different with the non-sliding scattering center. Therefore, it is a feature for the target recognition based on the micro-Doppler of the sliding scattering center.

If $R_{\mathrm{r}}=R_{\mathrm{t}}=0$ (this means that the target is flying toward the radar), then $f_{\mathrm{md}, 1}(t)=0$. There is no microDoppler, and no micro-Doppler effect caused by the sliding of $\mathrm{P}_{1}$.

\section{b) Micro-Doppler of $P_{2}$ and $P_{3}$}

Substituting (2), (6), (11), (25) into (28), then it can be derived that

$$
\begin{aligned}
& f_{\mathrm{md}, 2}(t)= \\
& \frac{k}{2 \pi} \frac{\mathrm{d}}{\mathrm{d} t}\left[b\left(\sin \theta_{\mathrm{r}}+\sin \theta_{\mathrm{t}} \cos \varphi_{\mathrm{t}}\right)+(l-h)\left(\cos \theta_{\mathrm{r}}+\cos \theta_{\mathrm{t}}\right)\right] .
\end{aligned}
$$

Substituting (2), (6), (11), (26) into (28), then it can be derived that

$$
\begin{aligned}
& f_{\mathrm{md}, 3}(t)= \\
& \frac{k}{2 \pi} \frac{\mathrm{d}}{\mathrm{d} t}\left[-b\left(\sin \theta_{\mathrm{r}}+\sin \theta_{\mathrm{t}} \cos \varphi_{\mathrm{t}}\right)+(l-h)\left(\cos \theta_{\mathrm{r}}+\cos \theta_{\mathrm{t}}\right)\right] . \\
& \text { So, }
\end{aligned}
$$

$$
f_{\mathrm{md}, 2}(t)+f_{\mathrm{md}, 3}(t)=2(l-h) \frac{k}{2 \pi} \frac{\mathrm{d}}{\mathrm{d} t}\left(\cos \theta_{\mathrm{r}}+\cos \theta_{\mathrm{t}}\right) .
$$

That is, $f_{\mathrm{md}, 2}(t)+f_{\mathrm{md}, 3}(t)$ has the same time-frequency curve with that of the micro-Doppler of the scattering point located on the bottom point $\mathrm{B}$.

To evaluate the influence of the sliding of $\mathrm{P}_{2}$, equation (31) can be rewritten as

$$
\begin{aligned}
& f_{\mathrm{md}, 2}(t)= \\
& \frac{k}{2 \pi} \frac{\mathrm{d}}{\mathrm{d} t}\left[b\left(\sin \theta_{\mathrm{r}}+\sin \theta_{\mathrm{t}} \cos \varphi_{\mathrm{t}}\right)+(l-h)\left(\cos \theta_{\mathrm{r}}+\cos \theta_{\mathrm{t}}\right)\right]= \\
& \frac{k}{2 \pi}\left\{\left[(l-h)-b \cot \theta_{\mathrm{r}}\right] \Lambda+\left[(l-h)-b \cot \theta_{\mathrm{t}} \cos \varphi_{\mathrm{t}}\right] \Pi+\sin \theta_{\mathrm{t}} \Sigma\right\} .
\end{aligned}
$$

In the same way, it can be obtained that

$f_{\mathrm{md}, 3}(t)=$

$\frac{k}{2 \pi} \frac{\mathrm{d}}{\mathrm{d} t}\left[-b\left(\sin \theta_{\mathrm{r}}+\sin \theta_{\mathrm{t}} \cos \varphi_{\mathrm{t}}\right)+(l-h)\left(\cos \theta_{\mathrm{r}}+\cos \theta_{\mathrm{t}}\right)\right]=$

$\frac{k}{2 \pi}\left\{\left[(l-h)+b \cot \theta_{\mathrm{r}}\right] \Lambda+\left[(l-h)+b \cot \theta_{\mathrm{t}} \cos \varphi_{\mathrm{t}}\right] \Pi+\sin \theta_{\mathrm{t}} \Sigma\right\}$

It can be seen that the micro-Doppler consists of two parts, one is caused by the size $(l-h)$, and the other is caused by $b$. They are sizes on two orthogonal dimensions, $Z$ and the lateral respectively. Therefore, the effect of the scattering center sliding is the shape change of the microDoppler distributions along with time.

When $\theta_{\mathrm{r}} \rightarrow \pi / 2$ and $\theta_{\mathrm{t}} \rightarrow \pi / 2$, it can be seen from (34), (35) that $f_{\mathrm{md}, 2}(t) \rightarrow f_{\mathrm{md}, 3}(t)$. This means that when the target is very near the point $\mathrm{O}$ in Fig. 1, the micro-Doppler of $\mathrm{P}_{2}$ and $\mathrm{P}_{3}$ are approaching the same one.

When $\theta_{\mathrm{r}} \rightarrow 0$ and $\theta_{\mathrm{t}} \rightarrow 0$, it can be seen from (34), (35) that $f_{\mathrm{md}, 2}(t) \rightarrow-f_{\mathrm{md}, 3}(t)$. This means that when the target is

$$
\begin{aligned}
& f_{\mathrm{md}, 1}(t)=\frac{1}{2 \pi} \frac{\mathrm{d}}{\mathrm{d} t}\left(-k \Delta r_{1}\right)=
\end{aligned}
$$

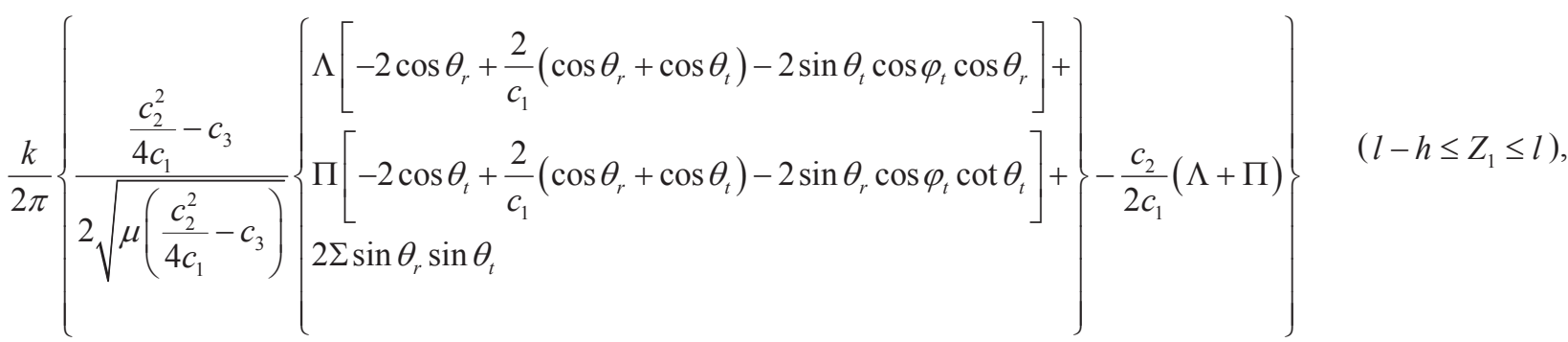

$$
\begin{aligned}
& \Lambda=\frac{\mathrm{d} \cos \theta_{\mathrm{r}}}{\mathrm{d} t}=-\frac{v^{2} t}{p^{3}}\left[R_{\mathrm{r}} \sin \alpha \sin \left(\omega t+\varphi_{0}\right)-v t \cos \alpha\right]+\frac{1}{p}\left[\omega R_{\mathrm{r}} \sin \alpha \cos \left(\omega t+\varphi_{0}\right)-v \cos \alpha\right] \text {, } \\
& \Pi=\frac{\mathrm{d} \cos \theta_{\mathrm{t}}}{\mathrm{d} t}=-\frac{v^{2} t}{q^{3}}\left[R_{\mathrm{t}} \sin \alpha \sin \left(\omega t+\varphi_{0}+\phi\right)+\left(z_{t}-v t\right) \cos \alpha\right]+\frac{1}{q}\left[\omega R_{\mathrm{t}} \sin \alpha \cos \left(\omega t+\varphi_{0}+\phi\right)-v \cos \alpha\right], \\
& \Sigma=\frac{\mathrm{d} \cos \varphi_{t}}{\mathrm{~d} t} \\
& =\frac{2 v^{2} t-v z_{\mathrm{t}}}{p q \sin \theta_{\mathrm{r}} \sin \theta_{\mathrm{t}}}-\left(\frac{\cot \theta_{\mathrm{t}}}{\sin ^{3} \theta_{\mathrm{r}}} \Lambda+\frac{\cot \theta_{\mathrm{r}}}{\sin ^{3} \theta_{\mathrm{t}}} \Pi\right)-\frac{R_{\mathrm{r}} R_{\mathrm{t}} \cos \phi-v t\left(z_{\mathrm{t}}-v t\right)}{\left(p q \sin \theta_{\mathrm{r}} \sin \theta_{\mathrm{t}}\right)^{2}}\left[v^{2} t \sin \theta_{\mathrm{r}} \sin \theta_{\mathrm{t}}\left(\frac{p}{q}+\frac{q}{p}\right)-p q\left(\sin \theta_{\mathrm{t}} \cot \theta_{\mathrm{r}} \Lambda+\sin \theta_{\mathrm{r}} \cot \theta_{\mathrm{t}} \Pi\right)\right] .
\end{aligned}
$$


far away from the point $\mathrm{O}$ in Fig. 1, the micro-Doppler of $\mathrm{P}_{2}$ and $\mathrm{P}_{3}$ are approaching the negative of each other.

When $\theta_{\mathrm{r}}=\theta_{\mathrm{t}} \rightarrow 0$, that is, the target is far away from the point $\mathrm{O}$ in Fig. 1 and the radar is mono-static, it can be seen from (29), (35) that the shape of the micro-Doppler curve of $\mathrm{P}_{1}$ and $\mathrm{P}_{3}$ are approaching to each other on the condition that $c_{1} \rightarrow 0$ and $a<l$.

When $R_{\mathrm{r}}=R_{\mathrm{t}}=0$, that is, the target is flying toward the radar, it can be seen that $f_{\mathrm{md}, 2}(t)=f_{\mathrm{md}, 3}(t)=0$. There are no micro-Doppler, and no micro-Doppler effect caused by the sliding of $\mathrm{P}_{2}$ and $\mathrm{P}_{3}$, too.

\section{Numerical Validations}

The analytical expressions of (29), (34), (35) can be validated by the simulated results based on the full-wave method of electromagnetic computation.

The parameters are set as follows. The wavelength is $0.03 \mathrm{~m} . a$ is $0.25 \mathrm{~m}, l$ is $1 \mathrm{~m}$, and $h$ is $1.25 \mathrm{~m}, c_{1}$ is $10^{-6}$. The velocity of the target is $1000 \mathrm{~m} / \mathrm{s}, \omega$ is $1 \mathrm{rad} / \mathrm{s}, \alpha$ is $0.1 \mathrm{rad}$, and $\varphi_{0}$ is zero. $R_{\mathrm{t}}$ and $R_{\mathrm{r}}$ are both $200 \mathrm{~km} . z_{\mathrm{t}}$ and $\varphi$ are both zeros.

Instantaneous micro-Doppler frequency distributions based on (29), (34), (35) are given in Fig. 3. Figure 3(a) gives the instantaneous micro-Doppler frequency distributions along with time from $-1000 \mathrm{~s}$ to $-975 \mathrm{~s}$, from $-50 \mathrm{~s}$ to $-25 \mathrm{~s}$, and from $-25 \mathrm{~s}$ to $0 \mathrm{~s}$. And Figure 3(b) gives the instantaneous micro-Doppler frequency distributions along with time from $975 \mathrm{~s}$ to $1000 \mathrm{~s}$, from $25 \mathrm{~s}$ to $50 \mathrm{~s}$, and from $0 \mathrm{~s}$ to $25 \mathrm{~s}$. For comparison with the non-sliding, the imaginary micro-Doppler of point $\mathrm{T}$ is given in the same figure.

From the first subplot of Fig. 3(a), it can be seen that the micro-Doppler of $\mathrm{P}_{1}$ and $\mathrm{P}_{3}$ are approaching each other and $f_{\mathrm{md}, 2}(t) \rightarrow-f_{\mathrm{md}, 3}(t)$ approximately, just the same as the analysis in Sec. 3 for small $\theta_{\mathrm{r}}$ and $\theta_{\mathrm{t}}$. And they are approaching the imaginary micro-Doppler of point $\mathrm{T}$ in the subplot for $\mathrm{P}_{1}$ and $\mathrm{P}_{3}$, which also can be seen from (29), (35).

In the second and the third subplots of Fig. 3(a), $\theta_{\mathrm{r}} \rightarrow \pi / 2$ and $\theta_{\mathrm{t}} \rightarrow \pi / 2$. In this situation, it can be seen from the subplots that $f_{\mathrm{md}, 2}(t) \rightarrow f_{\mathrm{md}, 3}(t)$, just the same as the analysis in Sec. 3. The micro-Doppler of $P_{1}$ is less than that of $\mathrm{T}$ due to scattering center sliding. In these two subplots, the micro-Doppler of $\mathrm{P}_{1}$ is to disappear gradually. This is because of the target approaching point $\mathrm{O}$.

When the target is far away from the radar, the sliding of $\mathrm{P}_{1}$ is not obvious. Compared with the imaginary micro-Doppler of point $T$, the influence of the sliding of $P_{1}$ is apparent when the target is approaching to the point $\mathrm{O}$. The value and the shape of the micro-Doppler distribution curve of $\mathrm{P}_{1}$ are influenced. The value becomes less, and the shape is distorted. For $\mathrm{P}_{2}$ and $\mathrm{P}_{3}$, the sliding of them influences the shape of the micro-Doppler distribution curve mainly when the target is far away from the radar. This means that
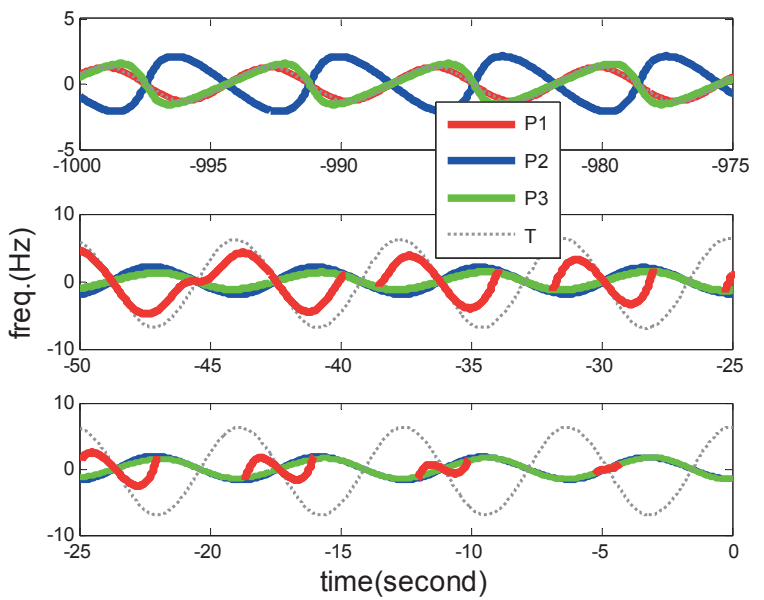

(a)
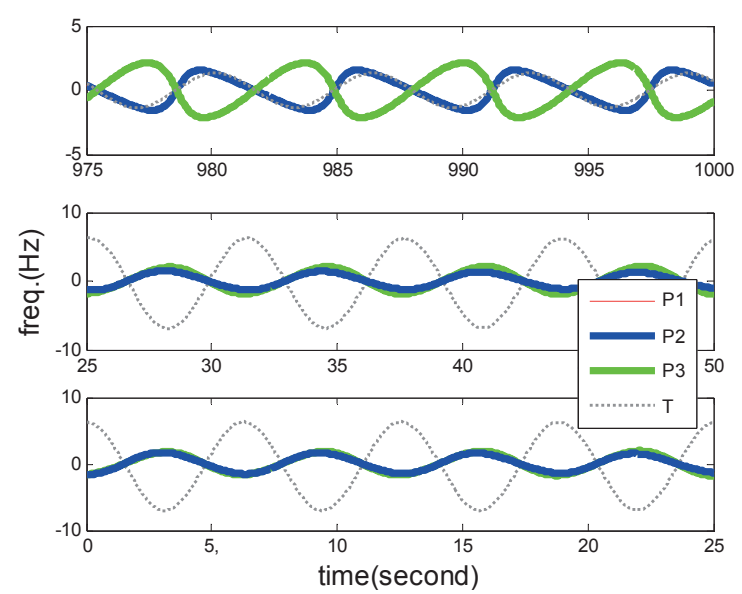

(b)

Fig. 3. Micro-Doppler of the target along with time $(v=1000 \mathrm{~m} / \mathrm{s})$.

the influence of the sliding is different for these two different types of the sliding scattering centers $\mathrm{P}_{1}$ and $\mathrm{P}_{2}\left(\mathrm{P}_{3}\right)$.

The characteristics of Fig. 3(b) are similar to Fig. 3(a) except that there's no micro-Doppler of $\mathrm{P}_{1}$ in Fig. 3(b). When $t>0$, the sliding scattering center $\mathrm{P}_{1}$ disappears.

For comparison, the simulated results of the microDoppler of the same situation are given. The simulated results of the micro-Doppler are based on the backscattering field results computed by the hybrid finite elementboundary integral-multilevel fast multi-pole algorithm (FE-BI-MLFMA) [20-22]. The time-frequency presentations (TFR) are given in Fig. 4 for the same time as Fig. 3. The TFR are obtained based on the short-time Fourier transform [23-25].

From Fig. 3 and Fig. 4, it can be seen that the results of Fig. 3 are consistent with the results of Fig. 4. Therefore, the analytical expressions of the micro-Doppler in (29), (34), (35) are validated. Note that Fig. 3 only gives the micro-Doppler while Fig. 4 gives the amplitude of the microDoppler frequency spectrum component.

The analytical expressions of the micro-Doppler of the sliding scattering centers are helpful to analyze the TFR 

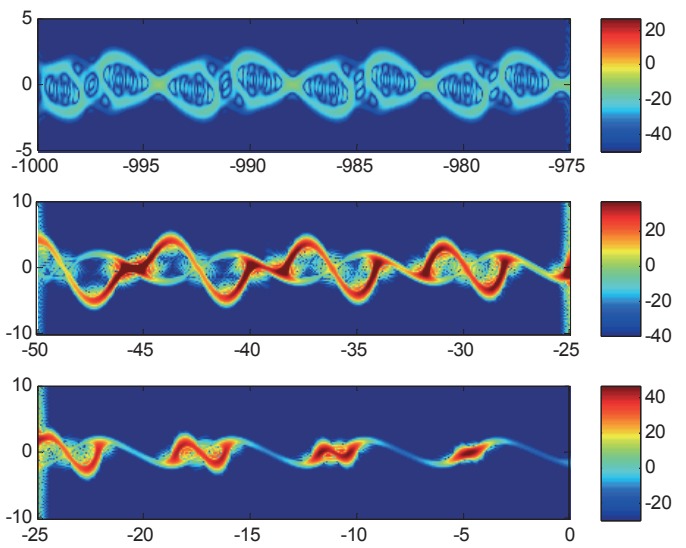

(a)
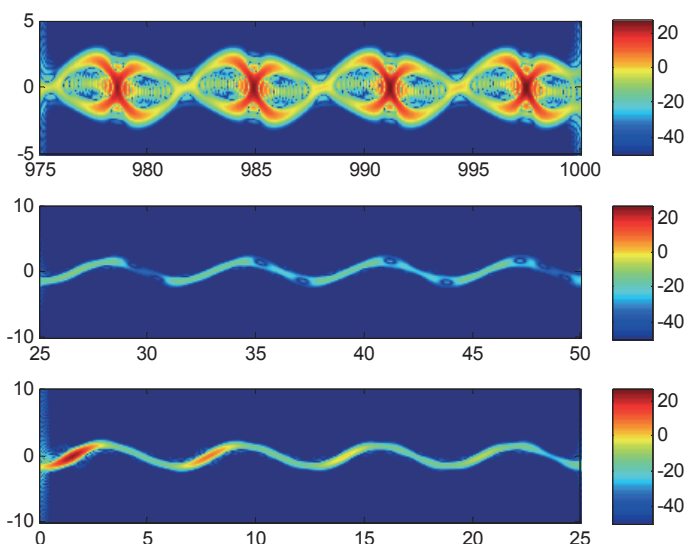

(b)

Fig. 4. TFR of the target (abscissa: time/second; vertical coordinate: frequency $/ \mathrm{Hz}$; amplitude: $\mathrm{dB}$ ).

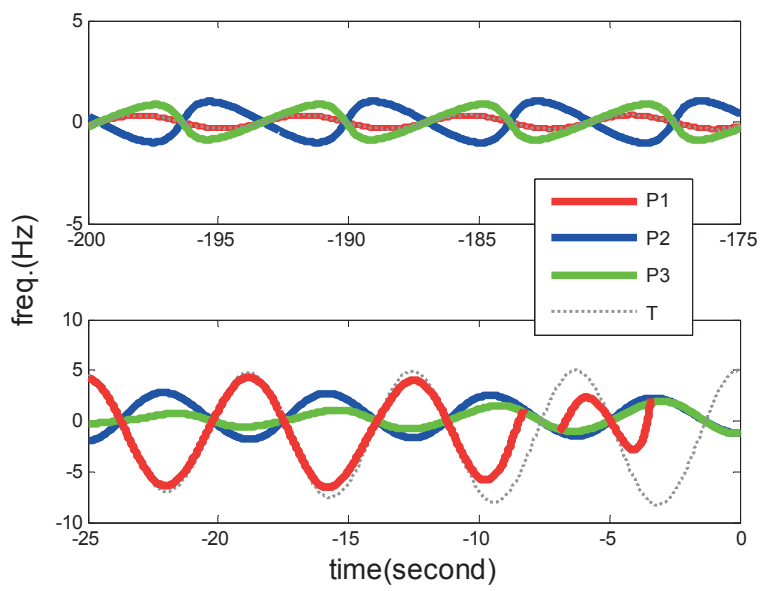

Fig. 5. Micro-Doppler of the target along with time $(v=5000 \mathrm{~m} / \mathrm{s})$.

of the target. For example, in the first subplot of Fig. 4(a), it can be known based on the analytical expressions that there are three curves for $\mathrm{P} 1, \mathrm{P} 2$ and $\mathrm{P} 3$ respectively actually. And the curves of P1 and P3 are near to each other and seem to be one, a distorted one. Therefore, there seems to be only two curves of micro-Doppler in the subplot, one is smooth, and the other is distorted.

As mentioned before, papers [18] have not considered the translation of the mass center. In this paper, to evaluate the influence of the translation velocity on the micro-Doppler of the sliding scattering centers, the numerical results based on the analytical expressions with $v=5000 \mathrm{~m} / \mathrm{s}$ are given in Fig. 5. And other parameters are the same as Fig. 3.

Note that the first subplot of Fig. 3 and the first subplot of Fig. 5 have the same starting position of the target, and the third subplot of Fig. 3 and the second subplot of Fig. 5 have the same ending position of the target. It can be seen that the characteristics of the micro-Doppler distribution curves are different in Fig. 3 and Fig. 5. Therefore, the analytical expressions with the translation influence in this paper are meaningful to the extension of the research in [18] for sliding scattering centers.

\section{Conclusions}

The analytical expressions of micro-Doppler of the sliding scattering centers are given based on the model of the extended streamlined targets for the bistatic radar. The sliding can make the micro-Doppler be less and distorted. And the sliding can make the TFR varying apparently along with time. The influence of the sliding is different for the two different types of the sliding scattering centers P1 and P2 (P3).The analytical expressions have been validated by the simulated results based on the full-wave method of the electromagnetic computation. The expressions are helpful to analyze the TFR of the extended streamlined target for the bistatic radar and to estimate the parameters of the target.

\section{Acknowledgments}

This work is supported by the National Natural Science Foundation of China (Grant No. 61471041) and by the Fundamental Research Funds for the Central Universities (FRF-TP-15-028A3).

\section{References}

[1] CHEN, V. C., LI, F., HO, S.S., et al. Analysis of micro-Doppler signatures. IEE Proceedings - Radar, Sonar and Navigation, 2003 vol. 150, no. 4, p. 271-276. DOI: 10.1049/ip-rsn:20030743

[2] CHEN, V. C. Micro-Doppler effect of micro-motion dynamics: a review. Proceedings of SPIE, 2003, vol. 5102, p. 240-249. DOI: $10.1117 / 12.488855$

[3] CHEN, V. C. The Micro-Doppler Effect in Radar. London (UK): Artech House, 2011. ISBN: 1608070573.

[4] CHEN, V. C., LI, F., HO, S. S., et al. Micro-Doppler effect in radar: Phenomenon, model, and simulation study. IEEE Transactions on Aerospace and Electronic Systems, 2006, vol. 42, no. 1, p. 2-21. DOI: 10.1109/TAES.2006.1603402

[5] RAM, S. S., CHRISTIANSON, C., KIM, Y., LING, H. Simulation and analysis of human micro-Dopplers in through-wall environments. IEEE Transactions on Geoscience and Remote Sensing, 2010, vol. 48, no. 4, p. 2015-2023. DOI: 10.1109/TGRS.2009.2037219 
[6] SURESH, P., THAYAPARAN, T., OBULESU, T., VENKATARAMANIAH, K. Extracting micro-Doppler radar signatures from rotating targets using Fourier-Bessel transform and time-frequency analysis. IEEE Transactions on Geoscience and Remote Sensing, 2014, vol. 52, no. 6, p. 3204-3210. DOI: 10.1109/TGRS 2013.2271706

[7] CHEN, V. C. Advances in applications of radar micro-Doppler signatures. In IEEE Conference on Antenna Measurements and Applications (CAMA). Antibes Juan-les-Pins, 2014, p. 1-4. DOI: 10.1109/ CAMA.2014.7003362

[8] VAN DORP, P., GROEN, F. C. A. Human walking estimation with radar. IEE Proceedings - Radar, Sonar and Navigation, 2003, vol. 150, no. 5, p. 356-365. DOI: 10.1049/ip-rsn:20030568

[9] MOLCHANOV, P., EGIAZARIAN, K., ASTOLA, J., et al. Classification of small UAVs and birds by micro-Doppler signatures. In Proceedings of the 10th European Radar Conference. Nuremberg, Germany, 2013, p. 172-175. DOI: $10.1017 / \mathrm{S} 1759078714000282$

[10] THAYAPARAN, T., ABROL, S., RISEBOROUGH, E., et al. Analysis of radar micro-Doppler signatures from experimental helicopter and human data. IET Radar, Sonar and Navigation, 2007, vol. 1, no. 4, p. 289-299. DOI: 10.1049/iet-rsn:20060103

[11] ZHANG, Q., YEO, T. S., TAN, H. S., et al. Imaging of a moving target with rotating parts based on the Hough transform. IEEE Transactions on Geoscience and Remote Sensing, 2007, vol. 42, no. 4, p. 291-299. DOI: 10.1109/TGRS.2007.907105

[12] KIM, Y., LING, H. Human activity classification based on microdoppler signatures using a support vector machine. IEEE Transactions on Geoscience and Remote Sensing, 2009, vol. 47, no. 5, p. 1328-1337. DOI: 10.1109/TGRS.2009.2012849

[13] AI, X., ZOU, X., LI, Y., et al. Bistatic scattering centres of cone-shaped targets and target length estimation. Science China Information Sciences, 2012, vol. 55, no. 12, p. 2888-2898. DOI: $10.1007 / \mathrm{s} 11432-012-4749-6$

[14] AI, X., ZOU, X., LIU, J., et al. Bistatic high range resolution profiles of precessing cone-shaped targets. IET Radar Sonar Navigation, 2013, vol. 7, no. 6, p. 615-622. DOI: 10.1049/iet-rsn.2012.0168

[15] QU, Q.Y., GUO, K.Y., SHENG, X.Q. An accurate bistatic scattering center model for extended cone-shaped targets. IEEE Transactions on Antennas and Propagation, 2014, vol. 62, no. 10, p. 5209-5218. DOI: 10.1109/TAP.2014.2342761

[16] QU, Q.Y., GUO, K.Y., SHENG, X.Q. Scattering centers induced by creeping waves on cone-shaped targets in bistatic mode. IEEE Transactions on Antennas and Propagation, 2015, vol. 63, no. 7, p. 3257-3262. DOI: 10.1109/TAP.2015.2424455

[17] MA, L., LIU, J., WANG, T., et al. Micro-Doppler characteristics of sliding scattering center on rotationally symmetric target.
Science China Information, 2011, vol. 54, no. 9, p. 1957-1967. DOI: $10.1007 / \mathrm{s} 11432-011-4254-3$

[18] GUO, K.Y., LI, Q.F., SHENG, X.Q., GASHINOVA, M. Sliding scattering center model for extended streamlined targets. Progress In Electromagnetics Research, 2013, vol. 139, p. 499-516. DOI: 10.2528/PIER13032111

[19] QU, Q.Y., GUO, K.Y., SHENG, X.Q. Applications of sliding scattering centers in feature extraction. In IEEE International Conference on Computational Electromagnetics (ICCEM). Hong Kong, 2015, p. 264-266. DOI: 10.1109/COMPEM.2015.7052628

[20] SHENG, X. Q., JIN, J. M., SONG, J. M., et al. On the formulation of the hybrid finite-element boundary-integral methods for 3D scattering. IEEE Transactions on Antennas and Propagation, 1998, vol. 46, no. 3, p. 303-311. DOI: 10.1109/8.662648

[21] SHENG, X. Q., YUNG, E. K.-N., CHAN, C.H., et al. Scattering from a large body with cracks and cavities by the fast and accurate finite-element boundary-integral method. IEEE Transactions on Antennas and Propagation, 2000, vol. 48, no. 8, p. 1153-1160. DOI: $10.1109 / 8.884482$

[22] GUO, K. Y., SHENG, X. Q. How to predict scattering and range profiles using complex targets with cavities. IEEE Transactions on Aerospace and Electronic Systems, 2011, vol. 47, no. 1, p. 155 to 165. DOI: 10.1109/TAES.2011.5705666

[23] CHEN, V. C., LING, H. Time-Frequency Transforms for Radar Imaging and Signal Analysis. Boston (US): Artech House Radar Library, Artech House, 2002. ISBN: 1-58053-288-8

[24] PAPANDREOU-SUPPAPPOLA, A. (ed.) Applications in TimeFrequency Signal Processing. $1^{\text {st }}$ ed. Boca Raton (US): CRC Press, 2003, p. 16-33. ISBN-10: 0849300657

[25] Time-Frequency Toolbox. Available at: http://tttb.nongnu.org/

\section{About the Author ...}

BO TANG received the B.S. degree in the Department of Physics from Nanjing University (NJU), Nanjing, China, in 2000; and the Ph.D. degree in the Institute of Electronics from the Chinese Academy of Sciences (CAS), Beijing, China, in 2005. He has been working in the School of Computer and Communication Engineering from the University of Science and Technology Beijing (USTB), Beijing, China, since 2005. His research interests include digital communications, microwave imaging, and radio frequency simulation. 